\title{
AN INTELLIGENT SYSTEM TO IMPROVE ATHLETE DEPRESSION AND EATING DISORDER USING ARTIFICIAL INTELLIGENCE AND BIG DATA ANALYSIS
}

\author{
Xuannuo Chen ${ }^{1}$ and Yu Sun ${ }^{2}$ \\ ${ }^{1}$ Linfield Christian, 31950 Pauba Road, Temecula, CA, 92592 \\ ${ }^{2}$ California State Polytechnic University, Pomona, CA, 91768
}

\begin{abstract}
The inspiration for the creation of this app stemmed from the deeply rooted history of eating disorders in sports, particularly in sports that emphasize appearance and muscularity which often includes gymnastics, figure skating, dance, and diving [1]. All three sports require rapid rotation in the air which automatically results in the necessity of a more stringent weight requirement. Eating disorders can also be aggravated by sports who focus on individual performances rather than team-oriented like basketball or soccer [5]. According to research, up to thirteen percent of all athletes have, or are currently suffering from a form of eating disorder such as anorexia [2] and bulimia [3]. In the National Collegiate Athletic Association, it is estimated that up to sixteen percent of male athletes and forty-five percent of female athletes have been diagnosed with an eating disorder.
\end{abstract}

\section{KEYWORDS}

Data Mining, Mobile APP, Machine Learning

\section{INTRODUCTION}

This app is meant for athletes to properly track their training schedule, as well as creating a proper ratio between training, eating, and resting. It allows athletes to log their daily activity level, as well as selecting their food intake and sleep hours [4]. It is applicable for athletes in all sports, as it does not contain specific restrictions. Additionally, this app contains key features such as a help page, which links all the important prevention hotlines in both English and Spanish. In creating this app, the goal is to control the levels of eating disorders in sports. Athletes are easily brainwashed by abusive coaches, and are given a falsified image of their bodies [6]. While outside factors such as these are uncontrollable, the athlete's own perception of themselves can be changed.

When first logging into the app, the sign-up screen appears first. New users have the ability to register themselves for the software, while returning users are able to log in using their email address and password. New users are asked to enter basic information about themselves, including their name and gender, which is stored in the profile page. Additionally, as an app aimed at decreasing the rates of eating disorders, athletes are asked for their current weight and height in order to determine any early signs of eating disorders. 
This app aims at reducing the rates of eating disorders through its three main components. When the athlete initially logs in, they are automatically directed to a fresh log page, where they can track their activity level, food intake, food quality, sleep hours, and sleep quality. Each category serves a specific purpose. In regards to activity level, food intake and hours of sleep should remain in a healthy ratio with their activity level. For example, two hours of sleep is insufficient for someone who has a five-hour practice period with high intensity. By entering their data, athletes are automatically promoted to reflect on their healthiness. Food intake and food quality have a stark difference fundamentally. Food intake suggests the amount of food consumed, which food quality measures the overall healthiness of the consumed food [7]. To put this into proper perspective, 600 grams of kale drastically differs from 600 grams of French fries. In addition, some foods are denser than others. 600 grams of kale can easily occupy a large salad bowl and is more than enough for one person, while 600 grams of sweet potatoes may only be one or two boiled potatoes, which only serves as a snack for many athletes, as sugar and carbohydrates are effective body fuels [8].

Similarly, sleep hours and sleep quality are vastly different. A person may lightly sleep for nine hours, and therefore feels fatigued the following day. Other times, athletes may sleep for only six hours, but become extremely energetic. Not only does the amount of sleep needed depend on the sport, but it also depends on the athlete. If only sleep hours were implemented, it would not be enough to personally measure the healthiness level of athletes. This is to prevent athletes from being pressured to over-sleeping, as it can result in unexpected tiredness. Normally, scientific research tends to suggest eight hours of sleep for young adults, while many may only need six hours.

Once the athlete submits their data for the day, they are then directed to the 'Dashboard' page, which tracks all of their past data. Users are permitted to access their past information by clicking on the tile. Having the ability to view past history allows the athletes to understand whether their eating and sleeping habits have improved. The athletes who consistently rank themselves as poor in regards to sleep quality rating can be identified as those who need sleep improvements. Seeing this, athletes can thereby apply for necessary help, including sleep treatments. Similarly, athletes can track their eating routine in proportion to their training hours and sleep levels. Oftentimes, those who deprive themselves of food do not have enough body energy to fuel for an entire session of practice. This can result in nausea, headaches, weak bodies, and in severe cases, injuries or fainting [9]. A special feature on the dashboard page is the randomly generated inspirational quotes from prominent athletes. These include Simone Biles, Michael Phelps, and many others. The purpose of this is simply to encourage struggling athletes on their path to success.

The last component of the app is the 'Help' page. In this page, athletes have access to a number of resources including websites and phone numbers to different hotlines. The National Suicide Hotline is placed on top in both English and Spanish as it is the most urgent and important. In the case of an emergency, athletes or those around them can rapidly access a series of phone numbers.

In general, eating disorders are directly related to abusive coaching methods. Many coaches have a tendency to blame the losses of athletes on their weight. A prime example of this is the experience of track runner Mary Cain. At seventeen years of age, Cain was one of the fastest runners in the United States, and qualified for the 2013 World Championships team as the youngest American in history. In the same year, Cain was signed to Nike's Oregon Project, the leading track and field program at the time, which was led by coach Alberto Salazar. As a coach who was only familiar with male athletes, Salazar became obsessed with Cain's weight, and revolved the entire training schedule on reducing weight. As a result of her consistent malnutrition, Cain's body began breaking down. Her bones had become fragile [15], which began 
to break easily, and she had also developed serious depression which resulted in actions of selfharm [10].

The abusive methods of coaching cannot be controlled by the opinions of the general public, as most of them are merely result-driven. Regulation should come from major organizations such as Safesport. The goal of this app is to manage what can be controlled, which is the athlete's perception of themselves. Through publishing this app, the hope is to see a reduction in the symptoms of eating disorders in users. This can be verified through changes made in the 'log' page. Improvements are justified through a change in behaviour, for example, consuming more healthy food and getting better sleep quality.

The rest of the paper is organized as follows: Section 2 gives the details on the challenges that we met during the experiment and designing the sample; Section 3 focuses on the details of our solutions corresponding to the challenges that we mentioned in Section 2; Section 4 presents the relevant details about the experiment we did, following by presenting the related work in Section 5. Finally, Section 6 gives the conclusion remarks, as well as pointing out the future work of this project.

\section{Challenges}

In order to build the tracking system, a few challenges have been identified as follows.

\subsection{Finding an Adequate Solution}

One major challenge that occurred throughout the process was finding an adequate solution to the original problem that was posed. Eating disorders are extremely overlooked in sports, meaning that minimal research has been done, and almost no preventative measures have been taken, especially in performance-based sports. In fact, it is basically impossible to eliminate eating disorders through one app, as the environment an athlete is in is the most outcome-defining factor. The most an app can do is to limit the potential of further developing eating disorders.

\subsection{Naming Each Label}

Additionally, it was difficult to find a proper wording for every label in the app. It was necessary to ensure that the words being used are not triggering under any circumstances. Requesting the user of the app to enter their weight upon registering can already cause insecurities among those who struggle with their body-image. Therefore, the selection of words must be done carefully and precisely. Words such as "fat" or "overweight" must be completely abandoned. This is because those who struggle with eating-disorders do not have an accurate perception of body-weight. Despite being possibly underweight, they may still believe the opposite, most likely due to the manipulative environment they practice in.

\subsection{Asking Athlete to Fill Logs}

In the end, it was determined that the most helpful method is asking their athlete to fill out daily logs that tracks their progress. This ensures that the app respects the user's privacy and sensitivity while promoting a healthier diet culture. Athletes can view their own progress by reviewing their past entries. For example, improvements are shown if the user historically indicates that they do not eat enough, and after two weeks, they start to eat more. 


\section{SOLUTION}

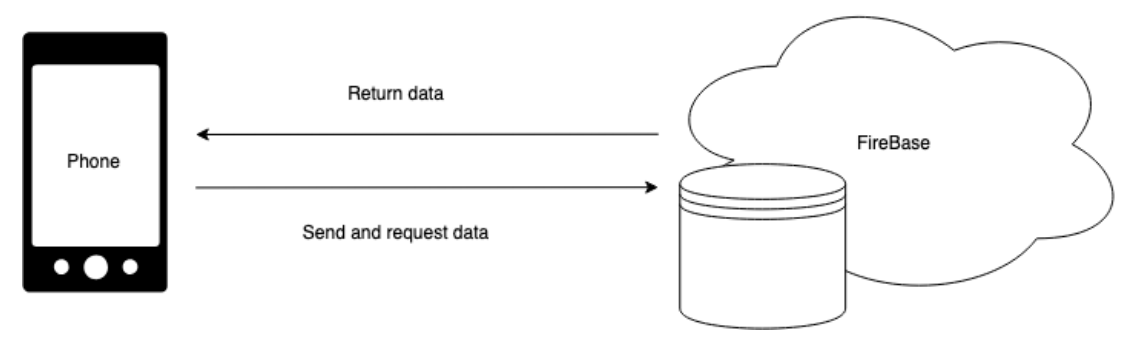

Figure 1. The overview of the project

Affly is a food health tracking app that helps users track the quality and quantity of their food and their sleeping quality. This is done by asking the user to input how much they eat and the quality of their food from a drop down based on how they feel. This subjective method of measuring and rating allows the user to reflect, think about and have an honest discussion with themselves about their habits.This method also avoids the difficulty of counting calories and tracking macro and micro nutrition [12]. Users are encouraged to add new data points daily and once the app has enough data it can show the users' habits over time and give users insights.

Affly utilities Google's Firebase tools to manage its users and their data. User creation is done within the Affly app itself only requiring an email for registration using Firebase's Authentication services. User data is stored using Firebase's Realtime Database. The information is stored using the users' unique ID and stores daily data using timestamps as keys under the users' unique ID [13].

Afflywas created using the flutter framework to allow easy development for both iOS and Android [14].

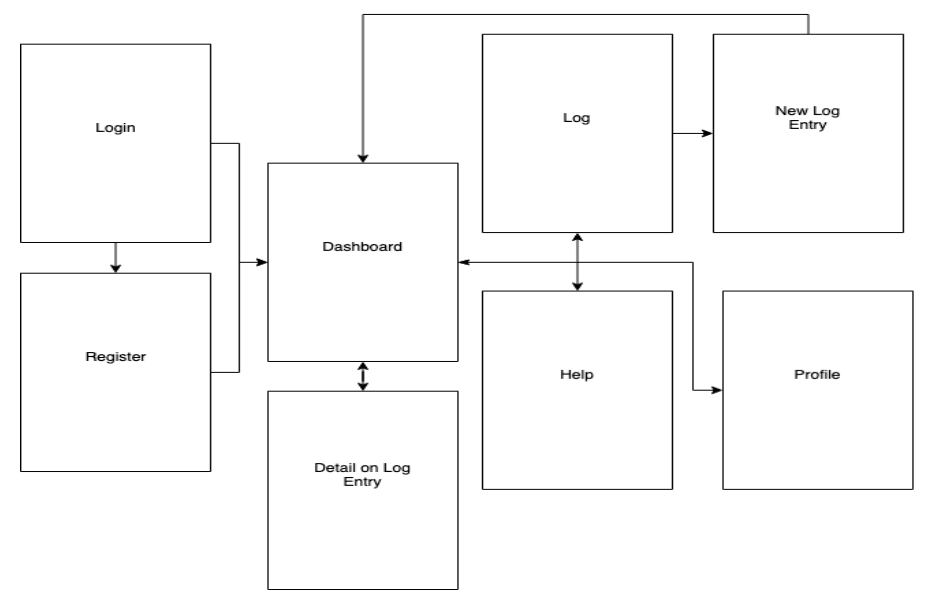

Figure 2. Basic process

Login:Allows the user to login.

Register: Allows the user to sign up and register.

Dashboard: Shows the user the latest insights of their health.

Log: Shows a list of log entries the user has entered. Pressing on a log entry lead to a detail page on the log entry. 
Computer Science \& Information Technology (CS \& IT)

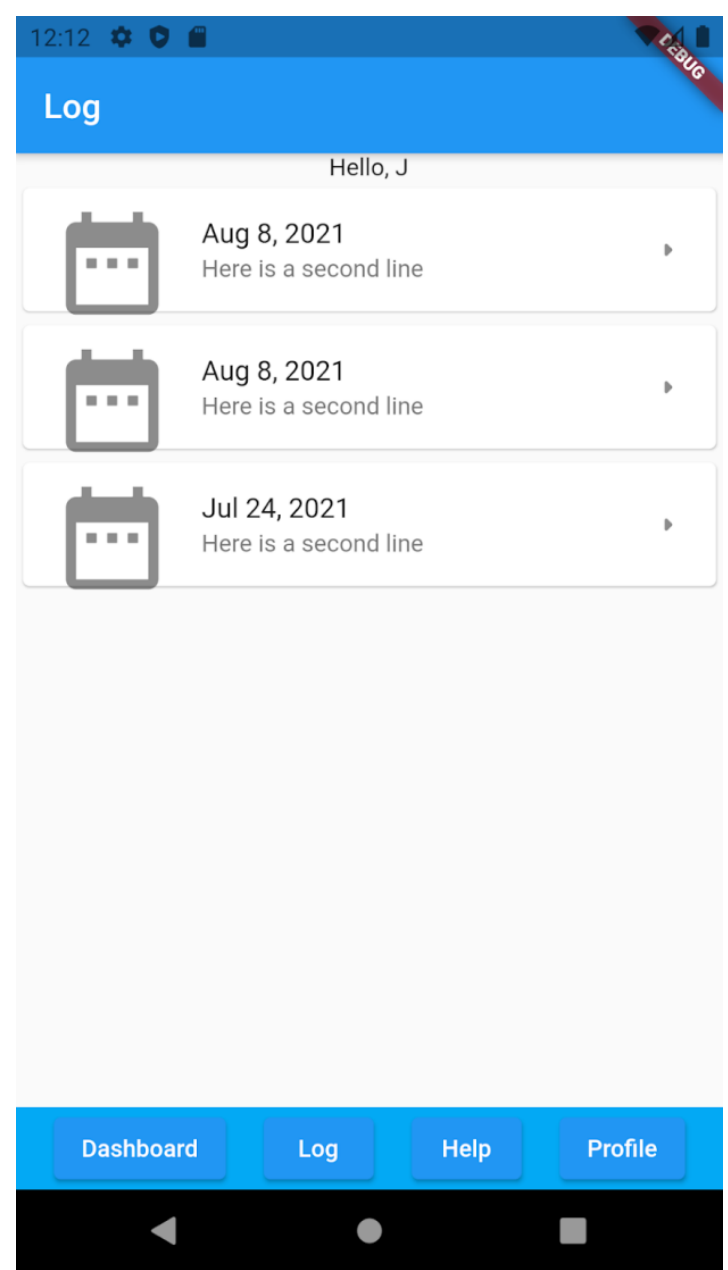

Figure 3. Screenshot of the App (1)

Log Entry Detail Page: Shows eatil about the log entry.

New Log Entry: This page allows the user to add a new entry. 


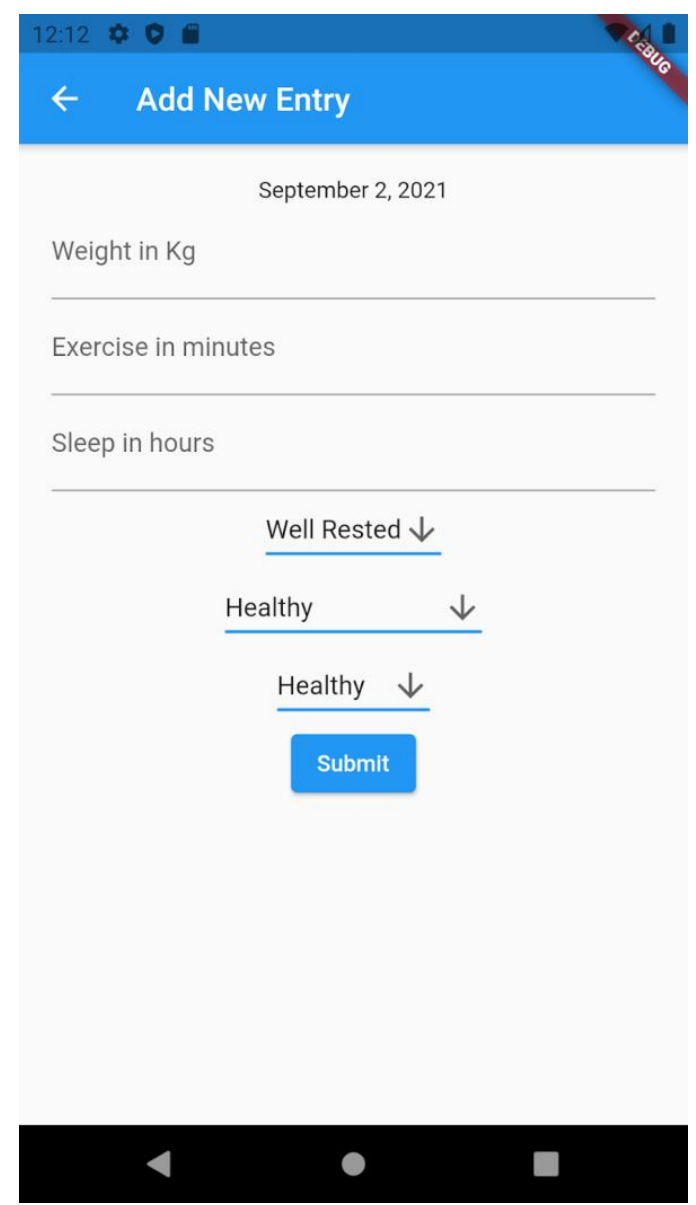

Figure 4. Screenshot of the App (2)

Help: This page lists resource users can contact for support.

Profile: This page shows the user info such as their email and profile name.

\section{EXPERIMENT}

\subsection{Experiment 1}

To prove the app works effectively and efficiently in the outdoor sports area, we ask 100 high school students who like to do sports like camping, walking trails, climbing mountains, and other outdoor sports, the Table shows the scores given by different types of sports lovers and the charts shows the ratio and distribution. We tested with 4 different types of sports and the test group is from different areas of Los Angeles so the group amount is big enough and group Diversity is guaranteed.

\begin{tabular}{|l|r|r|r|r|r|r|}
\hline & Total & Very Helpful & Helpful & Normal & Not help \\
\hline camping & 25 & 20 & 3 & 1 & 1 \\
\hline walking trails & 25 & 19 & 4 & 2 & 0 \\
\hline climbing mountains & 25 & 22 & 2 & 0 & 1 \\
\hline other outdoor sports & 25 & 20 & 5 & 0 & 0 \\
\hline
\end{tabular}

Figure 5. Result Matrix 


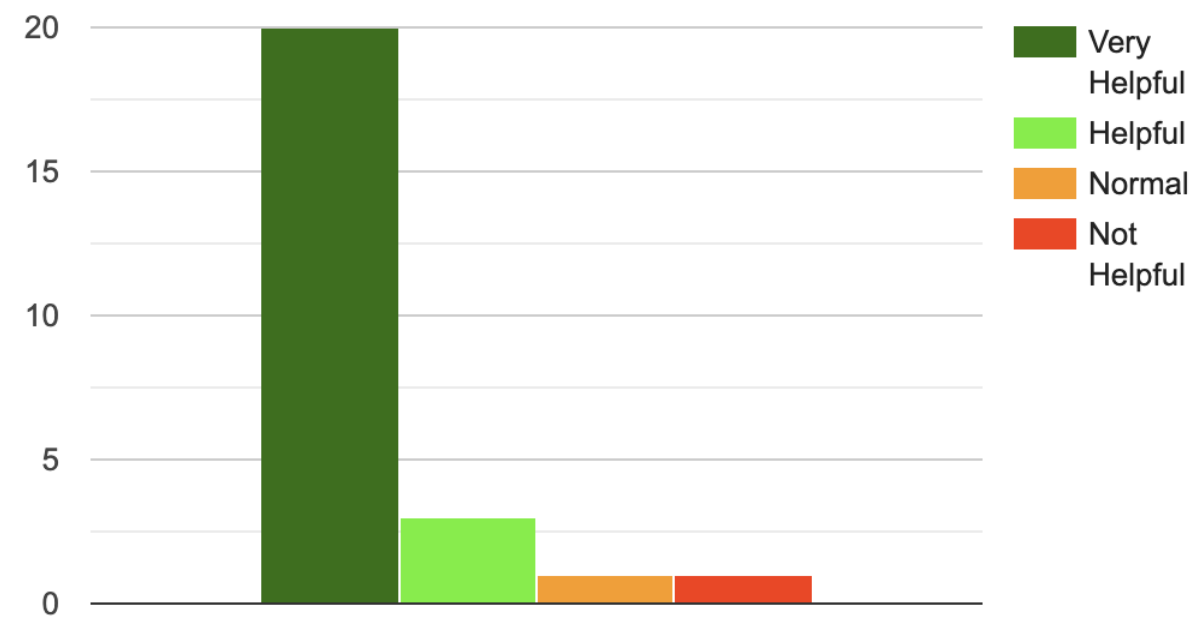

Figure 6. Score by Camping lover ratio

\section{Graph Title}

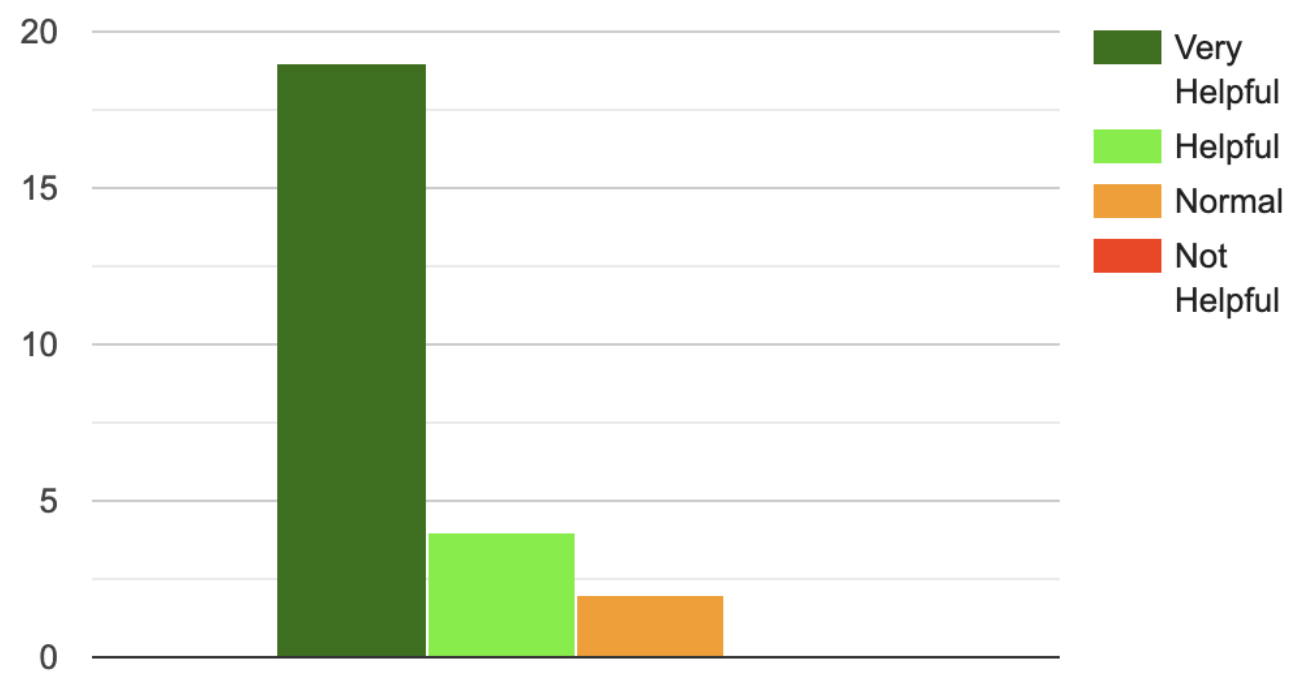

Figure 7. Score by walking trails ratio 


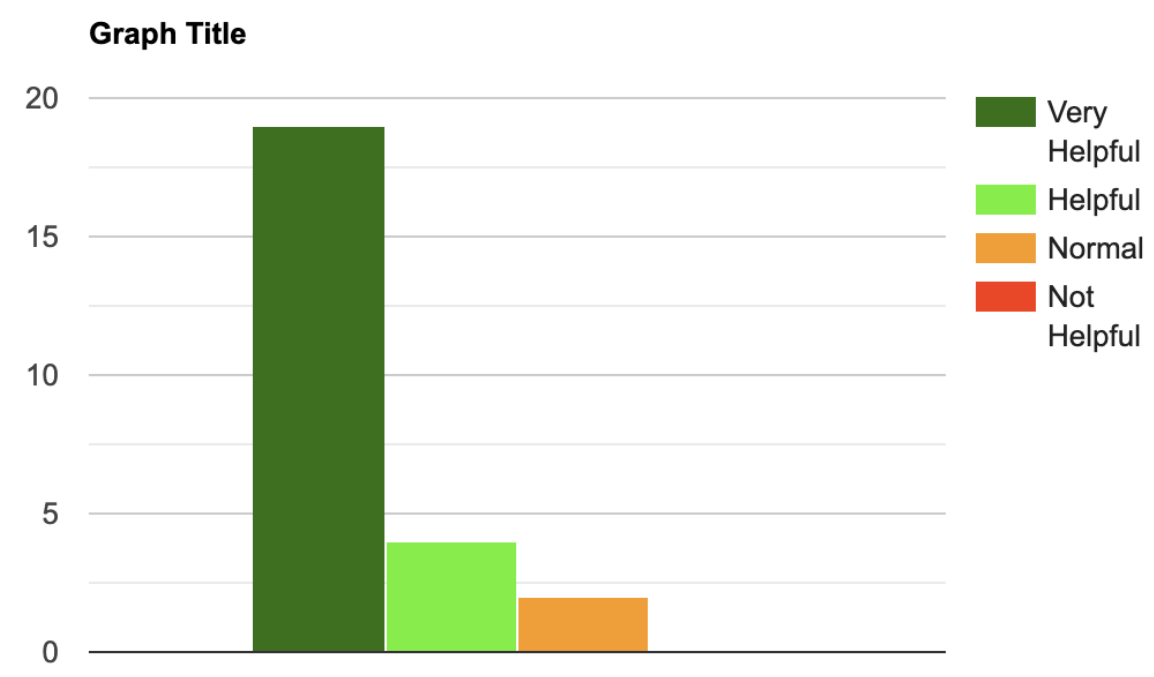

Figure 8 . Score by climbing mountains ratio

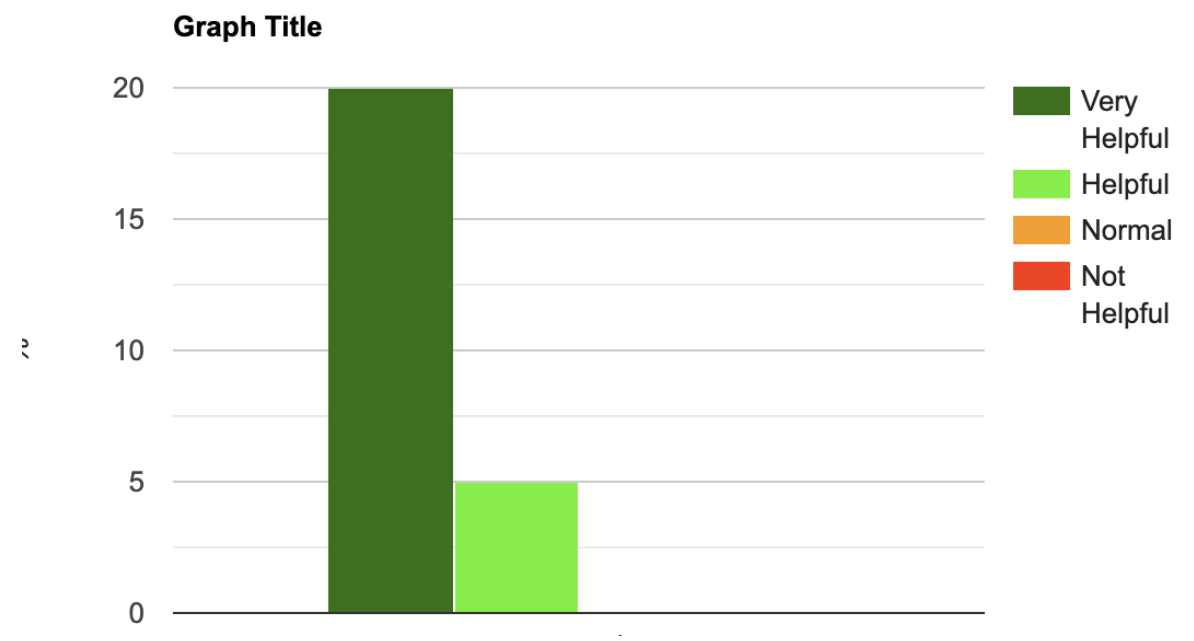

Figure 9. Score by other sports ratio

\subsection{Experiment 2}

For the second survey, we find 50 people who are working on training muscles and divide them into two groups. One group uses the app and the other one never uses it. We calculate the changes in the body fat rate of both groups. The two groups are following the same workout schedule and plan so It turns out people who work with the app reduce their body fat rate much faster than people who work without the app. The group works with the app to reduce their body rate average by $0.8 \%$, and the group working without the app reduces their body rate average by $0.3 \%$. 


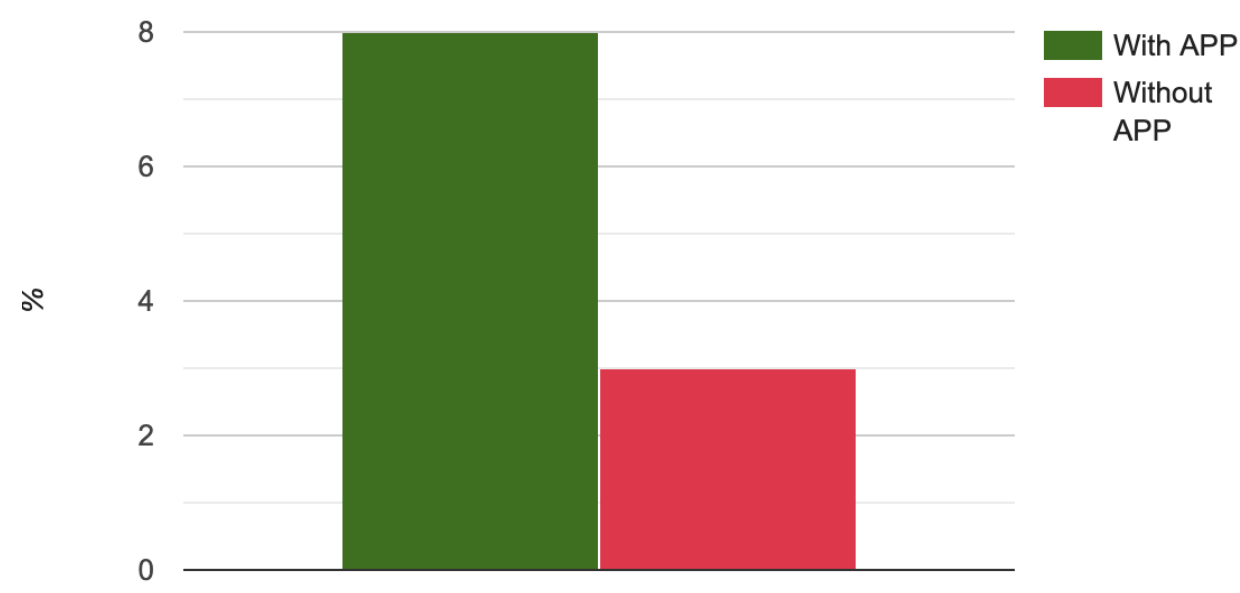

Figure 10. Survey result

Based on the above two experiments, we can prove that people who work with the app reduce the body fat rate much faster than people who work without the app. So we can consider the app to be effective and efficient.

\section{RELATED WORK}

A majority of last research done on eating disorders focuses on the treatments of eating disorders rather than the detection of earlier signs. This is because for a computer program, it is much easier to function through regulation. Most treatments will be similar for all users. On the other hand, early symptoms of eating disorders can vary, which makes it extra difficult to create an app that can accurately predict eating disorders.

\section{CONClusions}

The main component of this project is the construction of the application itself. To begin with, four parts of the app were proposed, including the "dashboard", "log", "help", and "profile" pages. The "log" and "help" pages are the most functional and crucial. In contrast, "dashboard" and "profile" are used for display purposes. Users will likely spend the longest time on "log" as they need to consistently track their daily progress. The dashboard indicates past entries and progress the user has made. Under normal circumstances, the user should not be constantly accessing the "help" page, however, if necessary, the "help" page is a valuable resource that can be potentially life-saving. Tests have been performed to test the overall functionality of the app. The "sign-up" and "log-in" pages are able to effortlessly upload users' information to the database to ensure that their history on the app does not become erased. The "log" page is able to accurately record the user's daily entries which are displayed in chronological order in the "dashboard".

While the app functions effectively and efficiently, there is plenty of room left for improvement. In the future, this app seeks to include automatic notifications. After tracking and analyzing all of the user's information, the app will automatically generate reports which will be returned to the user at their convenience. The user will have access to the app in the dashboard and will no longer be required to run their own analysis. This would be able to solve the app's largest issue: practicability [11]. Users who are already struggling with eating disorders will not have the time and energy to read about their past eating history. 


\section{REFERENCES}

[1] Hodge, Archibald, and Benjamin B. Warfield. Inspiration. Wipf and Stock Publishers, 2008.

[2] Bell, Rudolph M. Holy anorexia. University of Chicago Press, 2014.

[3] Pyle, Richard L., James E. Mitchell, and Elke D. Eckert. "Bulimia: a report of 34 cases." The Journal of Clinical Psychiatry (1981).

[4] Wardle, Jane, Kathryn Parmenter, and Jo Waller. "Nutrition knowledge and food intake." Appetite 34.3 (2000): 269-275.

[5] Polivy, Janet, and C. Peter Herman. "Causes of eating disorders." Annual review of psychology 53.1 (2002): 187-213.

[6] Satel, Sally, and Scott O. Lilienfeld. Brainwashed: The seductive appeal of mindless neuroscience. Basic Civitas Books, 2013.

[7] Kriss, Max. "THE FOOD CONSUMED, THE HEAT PRODUCTION, THE." Journal of Agricultural Research 40 (1930): 283.

[8] Burke, Louise M., et al. "Carbohydrates for training and competition." Journal of sports sciences 29.sup1 (2011): S17-S27.

[9] Stern, Robert Morris, Kenneth L. Koch, and Paul Andrews. Nausea: mechanisms and management. OUP USA, 2011.

[10] Pirlich, Matthias, et al. "The German hospital malnutrition study." Clinical nutrition 25.4 (2006): 563 572.

[11] Gioia, Dennis A. "Practicability, paradigms, and problems in stakeholder theorizing." Academy of Management Review 24.2 (1999): 228-232.

[12] Shenkin, Alan. "The key role of micronutrients." Clinical nutrition 25.1 (2006): 1-13.

[13] Klyne, Graham, and Chris Newman. Date and time on the internet: Timestamps. RFC 3339, July, 2002.

[14] Leshem, Shosh, and Vernon Trafford. "Overlooking the conceptual framework." Innovations in education and Teaching International 44.1 (2007): 93-105.

[15] Osaghae, Eghosa E. "Fragile states." Development in Practice 17.4-5 (2007): 691-699.

(C) 2021 By AIRCC Publishing Corporation. This article is published under the Creative Commons Attribution (CC BY) license. 